\title{
Tratamiento médico de la rinosinusitis crónica
}

\author{
Medical treatment of chronic rhinosinusitis. Literature review
}

David Jofré $\mathrm{P}^{1}$, Javiera Pardo J², Andrés Finkelstein $\mathrm{K}^{1}$.

\begin{abstract}
RESUMEN
La rinosinusitis crónica (RSC) es actualmente una de las patologías crónicas de mayor prevalencia en nuestra sociedad. Se distinguen dos formas clínicas: la RSC con pólipos (RSCCP) y la RSC sin pólipos (RSCSP). Es considerada, en términos generales, como una inflamación de la cavidad nasal y senos paranasales de una duración superior a 12 semanas.

En la actualidad, los posibles mecanismos fisiopatológicos involucrados ubican al componente inflamatorio como entidad central en su etiología. La relación entre inflamación y poliposis nasal es aún objeto de gran debate.

Existen distintos tratamientos médicos con evidencia científica de diferentes niveles de calidad, dentro de los cuales se encuentran antibióticos, corticoides, lavados nasales $y$ antileucotrienos.

El uso de macrólidos en bajas dosis y por períodos prolongados de tiempo surge como una eficaz alternativa tanto en el control de síntomas como de parámetros objetivos, principalmente en pacientes con RSCSP.

Este artículo efectúa una exposición respecto al tratamiento médico actualmente disponible, su eficacia y evidencia científica, tanto para la RSCP como para la RSCSP.

Palabras clave: Rinosinusitis crónica, poliposis nasal, antibióticos, corticoides, antileucotrienos, lavados nasales.
\end{abstract}

\begin{abstract}
Chronic rhinosinusitis (CRS) is currently one of the most prevalent chronic pathologies in Chile. Two forms are distinguishable: Polyp CRS and non-polyp CRS. CRS is condidered, generally speaking, an inflammation of the nasal cavities and paranasal sinuses lasting longer than 12 weeks. Current possible physiopathological mechanisms involved establish inflammation as a central entity in CRS etiology. The relationship between inflammation and nasal polyposis is still a matter of great debate. Several treatment options are available, supported by heterogeneous scientific evidence; among these are antibiotics, corticoids, nasal rinses and antileucotriens.

Prolonged treatment with low-dose macrolides treatment has become a good alternative, effectively controlling both symptoms and objective parameters, mainly in non-polyp CRS.

This article reviews the CRS medical treatment currently available, its efficacy and the scientific evidence supporting it, both for the polyp and non-polyp types.

Key words: Chronic rhinosinusitis, nasal polyposis, antibiotics, corticoids, antileukotrienes, nasal wash.
\end{abstract}

Médico Departamento de Otorrinolaringología, Hospital Clínico Pontificia Universidad Católica de Chile.

2 Médico Cirujano, Hospital Clínico Pontificia Universidad Católica de Chile. 


\section{INTRODUCCIÓN}

La rinosinusitis crónica (RSC) es reconocida como una de las patologías crónicas con mayor prevalencia en la actualidad. Se estima que $15,5 \%$ de la población en U.S.A. padece de RSC, la que es definida como inflamación sinusal de duración superior a 3 meses. Sin embargo, la prevalencia estimada disminuye a $2 \%$ cuando el diagnóstico es realizado por un médico ${ }^{1}$. De acuerdo a estas cifras existe una gran cantidad de pacientes involucrados, lo que genera una importante demanda de servicios y altos costos asociados. Esto refleja -en parte- la magnitud del problema.

En su evaluación y manejo participan diferentes niveles de atención y especialistas, dentro de los que destacan: médicos generales, pediatras, broncopulmonares, alergólogos e inmunólogos. Sin embargo, existe disparidad en los criterios diagnósticos a utilizar y respecto a los tratamientos que han demostrado ser de utilidad en estos pacientes. Es así como el médico otorrinolaringólogo debe recibir, con frecuencia, casos refractarios a tratamiento.

Según la Academia Americana de Otorrinolaringología y Cirugía de Cabeza y Cuello, se define como rinosinusitis a la inflamación de la cavidad nasal y senos paranasales. Ésta se caracteriza por la presencia de 20 más síntomas, entre los que necesariamente debe estar bloqueo/obstrucción/ congestión nasal, o la secreción nasal anterior/ posterior; acompañado de dolor/presión facial y/o pérdida del olfato. Además de la evaluación clínica, el diagnóstico puede ser realizado mediante endoscopía nasal, la que proporciona hallazgos sugerentes de rinosinusitis, como presencia de pólipos, descarga mucopurulenta desde el meato medio, y edema/obstrucción primaria de la mucosa de éste. Por otro lado, al evaluar mediante tomografía computarizada (TC) es posible encontrar alteraciones que también se incluyen en el diagnóstico, tales como cambios en la mucosa del complejo osteomeatal y/o senos paranasales ${ }^{1}$.

Es preciso señalar que ha sido descontinuado el uso de criterios mayores y menores debido a la mala correlación hallada en estudios prospecti$\operatorname{vos}^{2,3}$.

Se define la RSC como la persistencia de síntomas durante más de 12 semanas. Se considera la presencia de pólipos en el seno o meato medio como un subgrupo de esta entidad, excluyéndose de esta clasificación la enfermedad polipoídea de la cavidad nasal. Es así como se pueden establecer dos subgrupos: RSC con pólipos (RSCCP) y RSC sin pólipos (RSCSP).

No existe claridad respecto el por qué sólo algunos pacientes con RSC desarrollan pólipos. Sin embargo, estos pacientes se comportan de manera distinta y en su evolución tienen una alta tendencia a recurrir poscirugía.

El objetivo de este trabajo es realizar una revisión bibliográfica del tratamiento médico disponible tanto para RSCCP como para RRCSP y definir el tratamiento médico máximo para determinar con mayor claridad cuáles pacientes son candidatos a tratamiento quirúrgico. Los distintos niveles de evidencia de los estudios revisados se detallan en la Tabla 1.

Tabla 1. Niveles de evidencia científica

\begin{tabular}{|cl|}
\hline $\begin{array}{c}\text { Niveles de evidencia } \\
\text { científica }\end{array}$ & Graduación de los diseños de estudios* \\
\hline II-1 & $\begin{array}{l}\text { Evidencia a partir de ensayos clínicos aleatorios. } \\
\text { Evidencia a partir de ensayos clínicos no aleatorios. }\end{array}$ \\
II-2 & $\begin{array}{l}\text { Evidencia a partir de estudios de cohorte, casos y controles preferiblemente realizados } \\
\text { por más de un centro o grupo de investigación. } \\
\text { Evidencia a partir de comparaciones en el tiempo o entre sitios, con y sin la intervención, } \\
\text { podrían incluirse resultados espectaculares provenientes de estudios no aleatorios. } \\
\text { Opinión de expertos, basado en la experiencia clínica; estudios descriptivos o informes } \\
\text { de comités de expertos. }\end{array}$ \\
&
\end{tabular}

${ }^{*}$ Calidad de diseño y rigor científico de mayor (I) a menor (III). De: U.S. Preventive Task Force. Guide to clinical preventive services: An assessment of the effectiveness of 169 interventions. Baltimore: Williams and Wilkins, 1989. 


\section{ETIOPATOGENIA}

La etiología de la RSC aún no está clara. Clásicamente se creía que existía un fenómeno infeccioso subyacente que determinaba, entre otros mecanismos, obstrucción del ostium de drenaje y persistencia de agentes infecciosos. Sin embargo, en los últimos años se le ha reconocido al componente inflamatorio un rol importante, generado por varios factores en forma simultánea 0 independiente ${ }^{4}$.

Además, dentro de su etiología han sido estudiados otros elementos, como biofilms, hongos, rol del hueso (osteítis) y la presencia del súperantígeno estafilocócico.

Mecanismos inflamatorios, histopatológicos, celulares y moleculares diferencian a la RSCSP de la RSCCP. Muchos factores contribuyen a la activación de estos mecanismos, como disfunción del sistema mucociliar, infecciones virales, bacterianas o micóticas, alergias, edema de la mucosa, obstrucción causada por variaciones anatómicas de la cavidad nasal o senos paranasales y factores ambientales, entre otros.

Pese a la superposición de hallazgos encontrados entre RSCSP y RSCCP, existen diferencias importantes entre ambas. Las principales características histopatológicas de RSCSP son hiperplasia de células calciformes, engrosamiento de la membrana basal, hiperplasia de glándulas submucosas en el estroma e infiltrado crónico de células inflamatorias, con predominio de linfocitos y neutrófilos. Es así como el proceso inflamatorio crónico de la RSCSP muestra un predominio linfoplasmocítico, caracterizado por incremento de células secretoras (Tabla 2) ${ }^{5-12}$.

Por otra parte, la principal característica en la RSCCP es la presencia de eosinofilia con edema del estroma (Tabla 3) ${ }^{13-18}$.

En relación con el perfil de citoquinas y mediadores inflamatorios en RSCSP, éste es de tipo Th1 con aumento de mediadores relacionados con neutrófilos, linfocitos y células plasmáticas, como

Tabla 2. Células inflamatorias implicadas en rinosinusitis crónica sin pólipos

\begin{tabular}{|lll|}
\hline Autor & Células & Conclusión \\
\hline Bernardes $(2004)^{5}$ & Eosinófilos & Aumento de activación \\
Claeys $(2004)^{6}$ & Macrófagos & Mayor expresión de MMR y RNAm en RSCSP que RSCCP y controles \\
Kramer $(2004)^{7}$ & Mastocitos & MC participan en RSC \\
Rudack $(2004)^{8}$ & Neutrófilos & Dominan inflamación RSC \\
Polzehl $(2005)^{9}$ & Eosinófilos, MC & Infiltración diferenciada macrófagos, LC, LB RSCP y RSSP \\
Seiberling $(2005)^{10}$ & Eosinófilos & RSCCP nivel más alto que en RSCSP \\
Hafidh $(2006)^{11}$ & Eosinófilos & Aumento en RSC con hongos \\
Van Zele $(2006)^{12}$ & Eosinófilos & Mayor cantidad en RSCCP que en RSCSP \\
\hline
\end{tabular}

MMR: receptor de manosa de macrófagos. ARNm: ARN mensajero. MC: mastocitos. LC: leucotrienos. LB: linfocitos B.

Tabla 3. Células inflamatorias en rinosinusitis crónica con pólipos

\begin{tabular}{|lll|}
\hline Autor & Células & Conclusión \\
\hline Jankowski (1996) & Eosinófilos & $10 \%$ más de eosinófilos \\
Loessel $(2001)^{14}$ & Mastocitos & Sin diferencias v/s sin pólipos \\
Sobol $(2002)^{15}$ & Neutrófilos (en FQ) & Activación masiva \\
Shin $(2003)^{16}$ & Células epiteliales & Eosinófilos activados por citoquinas del epitelio nasal \\
Conley $(2006)^{17}$ & Receptores de LT & Interacción en 35\% para SAgs \\
Hao $(2006)^{18}$ & Linfocitos T & Relación inversa Cd4/Cd8 en pólipos v/s mucosa nasal \\
\hline
\end{tabular}

FQ: fibrosis quística. LT: leucotrienos. SAgs: superantígeno de Staphylococcus aureus. 
interferón (INF), TGF- $\beta$, IL-8 y mieloperoxidasa (MPO). Por el contrario, en la RSCCP predomina un perfil Th2, cuyos principales mediadores están relacionados con presencia de eosinófilos, como la proteína catiónica de eosinófilos (ECP), IL-5 e $\lg \mathrm{E}^{19}$.

La activación de mecanismos inflamatorios es mantenido por factores como infecciones, agentes irritantes y contaminantes (agentes físicos o químicos), que determinan cambios estructurales (histopatológicos) y funcionales (mediadores inflamatorios y citoquinas), los cuales requieren de una predisposición genética.

Estudios recientes han demostrado que en la patogénesis de la RSC juega un importante rol el reconocimiento de patógenos realizada por la inmunidad innata mediante células de la mucosa nasosinusal. La expresión de la proteína TLR9 (toll like receptor 9) está presente tanto en células epiteliales normales como en la RSC. Sin embargo, su nivel de expresión está reducido en RSCCP. Lo anterior sugiere que la respuesta inmune a patógenos vía TLR9 en la mucosa nasosinusal es inapropiada, pudiendo esto ser clave en el mecanismo inflamatorio de la RSC ${ }^{19}$.

La importancia de un enfoque orientado específicamente en la inflamación permite un manejo más racional de la patología, definir más claramente el 'tratamiento médico máximo' y evitar fracasos quirúrgicos.

\section{TRATAMIENTO MÉDICO EN RINOSINUSITIS CRÓNICA}

Existen distintos tipos de tratamientos para el manejo tanto de la RSCCP como de la RSCSP, por lo que un buen análisis de la evidencia actual permite definir aquellos pacientes candidatos a cirugía. Los distintos tratamientos evaluados se resumen en la Tabla 4.

\section{Tratamiento antibiótico}

El rol de las bacterias en RSC es muy debatido, teniendo mayor respaldo en rinosinusitis aguda y exacerbaciones en RSC; sin embargo, algunos consideran que la inflamación crónica es -en parte- causada por persistencia de bacterias. Existen en la literatura pocos estudios clínicos controlados y revisiones sistemáticas al respecto ${ }^{20}$.

Legent y cols (1994) ${ }^{21}$ estudiaron 251 pacientes con diagnóstico de RSC, comparándose el uso de amoxicilina/ácido clavulánico respecto a ciprofloxacino durante 9 días, no encontrando diferencias significativas entre ambos grupos, tanto en la mejoría clínica como en la erradicación bacteriológica. El ciprofloxacino mostró poseer una tasa de curación significativamente mayor que amoxicilina/ácido clavulánico.

Mc Nally y cols (1997) ${ }^{2}$ analizaron 200 casos de pacientes con diagnóstico de RSC, tratados durante 4 semanas con antibióticos orales, corticoides inhalados, lavados nasales y descongestionantes tópicos, evidenciando mejoría de signos y síntomas con el tratamiento médico.

Subramanian y cols $(2002)^{23}$ evaluaron mediante un estudio retrospectivo a 40 pacientes con diagnóstico de RSC, quienes fueron tratados durante un mes con betalactámicos, corticoides orales por un breve período, y terapia adyuvante (corticoides inhalados y lavados nasales). Los autores observaron mejoría significativa tanto en síntomas como en TC. Encontraron, además, que pacientes con antecedentes de poliposis nasal e

Tabla 4. Tratamiento médico en rinosinusitis crónica

\begin{tabular}{|l}
\hline Antibióticos: particularmente, los macrólidos \\
$:$ Corticoides: sistémicos e inhalados \\
$:$ Antihistamínicos \\
$:$ Descifungicos \\
$:$ Lavados nasales salinos \\
$:$ Inhibidores de bomba de protones \\
\hline
\end{tabular}


historia de cirugía de senos paranasales se asociaban a mayores tasas de recaída precoz sintomática. Sin embargo, se hace necesario realizar un estudio prospectivo comparado con un grupo placebo para avalar estos resultados.

Otro estudio comparó la efectividad y seguridad del uso de amoxicilina/ácido clavulánico con cefuroximo en el tratamiento de RSC y exacerbaciones agudas de RSC. Namyslowski y cols (2002) ${ }^{24}$ evaluaron la respuesta clínica y erradicación bacteriana, luego de 14 días de tratamiento antibiótico, no encontrando diferencias significativas entre ambos grupos. Sin embargo, la recurrencia clínica fue significativamente mayor en el grupo con cefuroximo.

De esta forma, es posible evidenciar mejoría clínica en algunos estudios; sin embargo, éstos carecen de grupos controles con placebo y poseen gran variabilidad en el tiempo de administración del antibiótico (entre 9 y 30 días), por lo que se hace necesario contar con estudios con mejor diseño para ratificar los resultados. La evidencia disponible se resume en la Tabla $5^{21-24}$.

Por otra parte, existe una buena concordancia entre la bacteriología hallada en muestras de meato medio e intrasinusal, de relevancia clínica ${ }^{25-29}$. Por consiguiente, el uso de cultivo de meato medio se considera de gran utilidad al momento de diagnosticar una reagudización en pacientes con RSC e indicar tratamiento antibiótico por corto plazo.

Dentro de lo novedoso del tratamiento antibiótico se ha postulado que el uso de macrólidos por períodos prolongados pudiese tener utilidad en el tratamiento de la RSCSP. Desde hace un tiempo se conoce que los macrólidos poseen efectos más allá de su acción antimicrobiana. En pacientes con panbronquiolitis difusa, una patología inflamatoria crónica de alta mortalidad, se observó que el uso de bajas dosis de eritromicina por períodos prolongados, aumentaba significativamente la sobrevida de estos pacientes ${ }^{30}$. Por otra parte, se sabe que Ios macrólidos no poseen acción bactericida sobre Pseudomona aeruginosa; sin embargo, son capaces de mejorar la función pulmonar, disminuir el uso de antibióticos y la necesidad de hospitalización en pacientes portadores de fibrosis quística, habitualmente colonizados por esta bacteria ${ }^{31}$. De esta forma Hirakata ${ }^{32}$ demostró que la eritromicina es capaz de inhibir la producción de exotoxina $A$, proteasa, elastasa y fosfolipasa $C$ producidas por Pseudomona aeruginosa, sugiriendo un potencial efecto inmunomodulador.

Los macrólidos tienen un efecto antiinflamatorio independiente de su acción antimicrobiana. Poseen la capacidad de acumularse en el intracelular de las células inflamatorias y desde ahí ejercer su efecto a distintos niveles. Modulan la producción de citoquinas, alteran la estructura y función de biofilms, reducen la expresión de moléculas de adhesión de leucocitos, aceleran la apoptosis de neutrófilos e inducen estrés oxidativo, mejoran el aclaramiento mucociliar y disminuyen las secreciones $^{33}$.

Tabla 5. Tratamiento antibiótico durante corto tiempo

\begin{tabular}{|c|c|c|c|c|c|}
\hline Estudio & Tipo AB & $\mathrm{N}^{0}$ & Tiempo & Efecto & $\begin{array}{l}\text { Nivel de } \\
\text { evidencia }\end{array}$ \\
\hline Namysloski $(2002)^{24}$ & AC.v/s cefuroximo & $206^{*}$ & 14 días & $\begin{array}{l}\text { Mejoría clínica, erradicación } \\
\text { bacteriológica recaída clínica }\end{array}$ & $n 0^{* *}$ \\
\hline Subramanian $(2002)^{23}$ & Betalactámicos & 40 & $4-6 \mathrm{sm}$ & Mejoría clínica y TC & $\| I^{* * *}$ \\
\hline Mac Nally $(1997)^{22}$ & Betalactámicos & 200 & $4 \mathrm{sm}$ & Respuesta clínica & $\| I^{\star \star \star *}$ \\
\hline Legent $(1994)^{21}$ & Ciprofloxacino v/s AC & 251 & 9 días & $\begin{array}{l}\text { Disminuye descarga nasal, respuesta } \\
\text { clínica y erradicación bacteriológica }\end{array}$ & $n 0^{\star *}$ \\
\hline
\end{tabular}

De: LUND VJ. Maximal medical therapy for chronic rhinosinusitis Otolaryngol Clin North Am 2005; 38: 1301-10.

AB: antibióticos. sm: semanas. AC: amoxicilina-ácido clavulánico. TC: tomografía computarizada.

* Incluye pacientes con exacerbaciones agudas y pacientes con rinosinusitis crónica.

** No existen pruebas que no muestren diferencias significativas entre los fármacos probados.

*** Nivel de evidencia III: pruebas de estudios descriptivos no experimentales como estudios comparativos, estudios de correlación y estudios de casos y controles. 
Su uso en RSCSP es conocido. Ya en 1996, Hashiba y $\operatorname{cols}^{34}$ demostraron respuesta de parámetros inflamatorios en adultos con RSCSP, después de 12 semanas de uso. Posteriormente, Cervin y cols (2000) $)^{33}$ encontraron que la respuesta a una terapia de 3 meses de duración se mantiene al año de observación.

Por otra parte, Walwork y cols $(2006)^{35}$ realizaron el primer estudio clínico controlado, demostrando ventajas significativas en parámetros subjetivos (puntaje clínico) y objetivos (test de sacarina, IL8 en lavado nasal, puntaje endoscópico) posterior a 12 semanas de uso con roxitromicina, principalmente en pacientes sin componente alérgico, demostrado por bajos niveles de IgE y de eosinófilos en secreción y mucosa nasal.

Es así como se han desarrollado criterios de selección de pacientes para uso de macrólidos (Tabla 6). Se recomienda, previo al inicio del tratamiento, solicitar Ig E plasmática (para descartar atopia), enzimas hepáticas y recuento de glóbulos blancos. Se debe monitorizar una posible resistencia bacteriana mediante cultivo nasal cada 3 meses y chequear efectos adversos repitiendo pruebas hepáticas y recuento de glóbulos blancos.

Desde el punto de vista práctico, se recomienda su empleo en el período de invierno y descansar durante los meses en que las infecciones son menos probables.

La claritromicina es el macrólido más estudiado en RSC, recomendándose su uso en dosis de $250 \mathrm{mg} / \mathrm{día} 0$ de roxitromicina $150 \mathrm{mg} / \mathrm{día}^{33}$.
Ragab y cols (2004) ${ }^{37}$ compararon la eficacia del tratamiento médico (lavado nasal, corticoides inhalados y macrólidos por 12 semanas) con cirugía endocópica nasal, en pacientes con RSCSP y RSCCP. Un total de 90 pacientes fueron sometidos a evaluaciones mediante escalas de síntomas, mediciones de óxido nítrico, rinomanometría acústica, tiempo de clearance de sacarina y endoscopía nasal. Estos fueron evaluados antes del inicio de tratamiento, a los 6 meses y al año de seguimiento. Los resultados obtenidos demuestran que tanto el tratamiento médico como el quirúrgico lograron mejoría significativa en los parámetros objetivos y subjetivos, sin diferencias significativas entre ambos grupos, con excepción del volumen nasal total en el grupo de RSCSP donde se observaron mayores cambios al ser tratados con cirugía. Los autores concluyeron que la terapia de la RSC, con y sin pólipos, debe iniciarse con el tratamiento médico máximo disponible, el que incluye uso de macrólidos durante 3 meses, lavados nasales y corticoides inhalados. La cirugía debe reservarse sólo en los casos de rebeldía al tratamiento médico. Por otra parte, la presencia de pólipos nasales no es un factor de mal pronóstico al evaluar eficacia, tanto del tratamiento médico como quirúrgico. Los estudios realizados se resumen en la Tabla $7^{34-37}$.

\section{Corticoides}

Los corticoides sistémicos e inhalados son ampliamente usados en todo el mundo en RSCSP y RSCCP. Su acción se debe a la activación de

Tabla 6. Selección de pacientes para uso de macrólidos

\begin{tabular}{|ll|}
\hline A favor & En contra \\
\hline IgE sérica normal & IgE elevada \\
No alérgico & Alérgico \\
Cultivo negativo & Cultivo positivo $\left(1^{\circ}\right.$ tto específico $)$ \\
Corticoide nasal no efectivo & Corticoide nasal efectivo \\
SP: descarga posterior, dolor facial, cefalea & SP: rinorrea serosa, estornudos \\
& Disquinesia ciliar primaria \\
& Sinusitis alérgica fúngica \\
\hline
\end{tabular}

De: Cervin A, Wallwork $B$. Anti-inflammatory effects of macrolide antibiotics in the treatment of chronic rhinosinusitis. Otolaryngol Clin North Am 2005; 38: 1339-50.

IgE: inmunoglobulina E. SP: síntomas principales. tto: tratamiento. 
Tabla 7. Tratamiento antibiótico durante tiempo prolongado

\begin{tabular}{|llclc|}
\hline Estudio & Tipo AB & Tiempo & Efecto & Nivel de evidencia \\
\hline Nishi (1995) & Claritromicina & $3 \mathrm{~m}$ & decrece exacerbación & III \\
Hashiba (1996) & Claritromicina & $3 \mathrm{~m}$ & mejoría clínica 70\% & III \\
Ragab (2004) $)^{37}$ & Eritromicina & $3 \mathrm{~m}$ & mejoran puntajes clínicos, Ib y CEF & Ib \\
Wallwork (2006) & Eritromicina & $3 \mathrm{~m}$ & mejoría global & Ib \\
\hline
\end{tabular}

AB: antibiótico. m: meses. Ib: laboratorio. CEF: cirugía endoscópica funcional.

receptores intracelulares de glucocorticoides. Los principales efectos en la mucosa nasal y en pólipos son por reducción de la infiltración eosinofílica y la secreción de citoquinas quimiotácticas ${ }^{1,20}$.

\section{A. Corticoides sistémicos}

Para evaluar los efectos de los corticoides sistémicos en pacientes con poliposis nasal, Van Camp y cols $(1994)^{38}$ diseñaron un estudio en el que incluyeron 25 pacientes con diagnóstico de poliposis nasal, a quienes trataron durante 4 días con prednisolona $60 \mathrm{mg}$ en dosis decrecientes y evaluaron cambios en la sintomatología, la rinoscopía anterior y la TC de cavidades paranasales. Encontraron que el $72 \%$ de los pacientes mostraba disminución subjetiva de síntomas, pero sólo $52 \%$ evidenciaba una mejoría clara en la TC. Por otra parte, la eficacia terapéutica fue mayor en el grupo de pacientes con intolerancia a la aspirina y menor en el grupo de alérgicos. Por lo anterior plantearon que los corticoides sistémicos eran útiles como terapia preoperatoria para facilitar la cirugía de pólipos. Concluyeron recomendando reservar su uso para estos casos.

Damm y cols (1999) ${ }^{39}$ evaluaron la eficacia de la terapia esteroidal combinada (budesonida intranasal y fluocortolone) durante 20 días en 20 pacientes con RSCCP severa, mediante cuestionarios de síntomas y resonancia nuclear magnética
(RNM). Observaron una reducción significativa (mayor al 30\%) en el 50\% de los hallazgos en la RNM y disminución de síntomas en el $80 \%$ de los pacientes, reafirmando que su mayor indicación es como tratamiento preoperatorio, facilitando la cirugía endoscópica del seno.

Benítez y cols $(2006)^{40}$ realizaron un estudio en 84 pacientes con poliposis nasal severa, evaluando el efecto de prednisona oral administrada durante 2 semanas, seguido de budesonida intranasal por 12 semanas, en comparación con placebo. Observaron que los corticoides orales producían una mejoría significativa de los síntomas, tamaño de los pólipos, flujo nasal y resultados en la TC comparado con el grupo no tratado, y que estos efectos eran mantenidos por budesonida intranasal.

Hissaria y cols (2006) ${ }^{41}$ administraron 50 mg/día de prednisona oral durante 14 días en 20 pacientes con RSCCP y lo compararon con placebo, encontrando diferencias significativas en disminución de síntomas, reducción del tamaño de los pólipos -evaluado por endoscopía nasal y RNM- comparado con el grupo control, concluyendo que es un tratamiento seguro y efectivo en la poliposis nasal sintomática.

De estos estudios se puede concluir que los corticoides orales son de utilidad en el tratamiento de pacientes con RSCCP. Los hallazgos obtenidos se resumen en Tabla $8^{38-41}$.

Tabla 8. Corticoides sistémicos en rinosinusitis crónica con pólipos

\begin{tabular}{|llccc|}
\hline Estudio & Droga/Dosis & Tiempo & Efectos & Nivel de evidencia \\
\hline Van Camp (1994) & Prednisona $(60 \mathrm{mg})$ & $2 \mathrm{sm}$ & sí & III \\
Damm $(1999)^{31}$ & Budesonida + fluocortolone & $?$ & sí & III \\
Benitez $(2006)^{32}$ & Prednisona + budesonida & $2 \mathrm{sm}$ & sí & Ib \\
Izaría $(2006)^{33}$ & Prednisolona $(50 \mathrm{mg})$ & $2 \mathrm{sm}$ & sí & Ib \\
\hline
\end{tabular}

sm: semanas. Ib: laboratorio. 
En contraste con lo anterior, no existen estudios clínicos controlados que demuestren la eficacia de corticoides orales en RSCSP20,42.

\section{B. Corticoides inhalados}

Los corticoides inhalados poseen múltiples efectos antiinflamatorios, entre ellos el reducir la producción de citoquinas proinflamatorias, y disminuir la llegada de células inflamatorias, mejorando así la obstrucción nasal. Son sus propiedades antiinflamatorias las que generan el alivio de síntomas asociados a la inflamación: congestión nasal, dolor facial y obstrucción nasal.

La administración de corticoides inhalados por largo tiempo es segura, ya que no produce atrofia de la mucosa u otro cambio histológico y mantiene la función ciliar o clearance mucociliar. Su uso no se ha asociado a efectos adversos sistémicos, como supresión del eje hipotálamo-hipófisisadrenal, impedimento del crecimiento en niños 0 alteración del metabolismo óseo, pudiendo exacerbar la osteoporosis. Sin embargo, su empleo ha sido relacionado con efectos adversos locales: epistaxis, irritación de nariz o garganta, sequedad local, cefalea, faringitis y costras nasales ${ }^{42}$.

2.B.1. Corticoides inhalados en rinosinusitis crónica con pólipos

Keith y cols $(2000)^{43}$ evaluaron, mediante un estudio clínico controlado multicéntrico, la efectividad de la fluticasona (400 $\mu \mathrm{gr}$ ) aplicada durante 12 semanas, comparada con placebo, en pacientes con RSCCP. Encontraron una mejoría significativa en la reducción del tamaño de pólipos, disminución de obstrucción nasal y mejoría del flujo inspiratorio máximo nasal en el grupo con fluticasona. Señalan que la epistaxis fue la complicación más frecuente en este grupo.
Pentilla y cols $(2000)^{44}$ realizaron un estudio para evaluar la efectividad y tolerabilidad de fluticasona (400 $\mu \mathrm{gr})$ al ser administrada durante 12 semanas, una o dos veces al día, en comparación con placebo, encontrando que el uso de una doble dosis diaria produce mejoría significativa del tamaño de los pólipos, síntomas de rinitis, flujo inspiratorio máximo nasal. Concluyen que una doble dosis de fluticasona es significativamente más efectiva que la dosis única y el placebo. Small y cols $(2005)^{45}$ realizaron un estudio multinacional, en el que incluyeron 354 pacientes y evaluaron la eficacia y seguridad de fluorato de mometasona (200 $\mu \mathrm{gr}$ ) en dosis única o doble al día, mostrando mejoría significativa en reducción del tamaño de pólipos, recuperación en la pérdida olfatoria, rinorrea anterior, descarga posterior y congestión/obstrucción nasal al compararlo con placebo, disminuyendo 0 retardando la necesidad de cirugía.

Stjärne y cols $(2006)^{46}$ diseñaron un estudio multicéntrico para evaluar en 310 pacientes la efectividad de mometasona (200 $\mu \mathrm{gr})$ en dosis diaria única o doble comparado con placebo. Luego de 4 meses de uso demostraron una mejoría estadísticamente significativa en reducir el tamaño de los pólipos, congestión y obstrucción nasal.

Con estos resultados se puede concluir que el uso de corticoides inhalados es una terapia segura y efectiva en el tratamiento de la RSCCP (Tabla 9) ${ }^{43-46}$.

2.B.2. Corticoides inhalados en rinosinusitis crónica sin pólipos.

Cuenant y cols (1986) ${ }^{47}$ estudiaron la efectividad de la irrigación con tixocortol más

Tabla 9. Corticoides inhalados en rinosinusitis crónica con pólipos

\begin{tabular}{|llclcc|}
\hline Estudio & Droga & Tiempo & Clínica & Nivel de evidencia \\
\hline Keith $(2000)^{35}$ & Fluticasona & $12 \mathrm{sm}$ & Mejoría subjetiva y objetiva & NS & lb \\
Pentilla $(2000)^{36}$ & Fluticasona & $12 \mathrm{sm}$ & Mejorías nasales y olfato & sí & lb \\
Small $(2005)^{37}$ & Mometasona & $16 \mathrm{sm}$ & Mejoría de obstrucción y olfato & sí & lb \\
Stjarne $(2006)^{38}$ & Mometasona & $16 \mathrm{sm}$ & Mejora objetiva & sí & Ib \\
\hline
\end{tabular}

sm: semanas. lb: laboratorio. 
neomicina en comparación con uso exclusivo de neomicina en el tratamiento de RSCSP en 60 pacientes, obteniendo mejoría significativa de la obstrucción nasal en el grupo de tixocortol más neomicina tras 11 días de tratamiento.

Ese mismo año, Sykes y cols $^{48}$ realizaron un estudio clínico controlado en 50 pacientes con diagnóstico de RSC mucopurulenta, para evaluar efectividad del tratamiento con dexametasona, tramazolina y neomicina comparado con igual terapia sin antibiótico. Encontraron una reducción de la respuesta inflamatoria y congestión nasal sin necesidad de tratamiento antibiótico, postulando que probablemente el efecto se debería a mecanismos que permiten recuperar el clearance mucociliar.

Para evaluar el uso de propionato de fluticasona en spray, Parikh y cols (2001) ${ }^{49}$ realizaron un estudio clínico durante 4 meses en pacientes con diagnóstico de RSC, evaluaron puntajes de síntomas, puntajes endoscópicos, rinomanometría acústica entre otros. Encontraron que no existían diferencias al compararlo con placebo. Estos autores hallaron que el uso de fluticasona en spray no aumenta el riesgo de infección y provoca dow-regulation en la expresión de citoquinas inflamatorias.

Lavigne y cols (2002) ${ }^{50}$ evaluaron la eficacia de budesonida (256 $\mu \mathrm{gr}$ ) en 26 pacientes con diagnóstico de RSCSP quienes tras ser operados persistieron con síntomas, tales como rinorrea o presión/dolor facial. El corticoide fue administrado mediante un dispositivo de intubación (tubo de sinusotomía antromaxilar) durante 3 semanas, encontrando mejoría en escala de síntomas, por lo que sería un tratamiento útil en el control de la RSCSP poscirugía.

Lund y cols (2004) $)^{51}$ investigaron la eficacia de budesonida (128 $\mu \mathrm{gr}$ ) en spray nasal en el tratamiento de la RSCSP durante 20 semanas. Encontraron mejoría en escala de síntomas, descarga y congestión nasal, anosmia y aumento del flujo inspiratorio máximo nasal en pacientes que usaron budesonida comparado con placebo, concluyendo que es un tratamiento efectivo y bien tolerado para el manejo de estos pacientes. Posteriormente este mismo autor (2008) ${ }^{34}$ realizó una revisión sistemática para evaluar la eficacia del tratamiento sintomático con corticoides intranasales en pacientes adultos y pediátricos con rinosinusitis aguda, recurrente 0 crónica. Todos los artículos incluidos eran estudios clínicos controlados que comparaban el uso de corticoides inhalados con placebo, ya sea monoterapia 0 terapia adyuvante. Su conclusión fue que los corticoides inhalados disminuyen el bloqueo nasal, descarga purulenta, dolor facial y cefalea, reduciendo la inflamación local y mejorando el drenaje (Tabla 10) ${ }^{47-51}$.

Los corticoides inhalados son seguros, efectivos, y no aumentan el riesgo de infección bacteriana 0 recurrencia. Como terapia adyuvante a los antibióticos en pacientes con rinosinusitis aguda, recurrente 0 exacerbación de RSC muestran mayor mejoría sintomática que el tratamiento antibiótico aislado ${ }^{42}$.

De este modo los procesos inflamatorios son objetivos terapéuticos clínicamente importantes en

Tabla 10. Corticoides inhalados en rinosinusitis crónica sin pólipos

\begin{tabular}{|llclc|}
\hline Estudio & Droga & Tiempo & Efecto en síntomas & Nivel de evidencia \\
\hline Cuenant $(1986)^{39}$ & Tixocortol (irrigación) & 11 días & Mejoría significativa & Ib \\
Sykes $(1986)^{40}$ & Dexametasona más tramazoline & $4 \mathrm{sm}$ & Mejoría síntomas & Il \\
Parikh $(2001)^{41}$ & Fluticasona & $16 \mathrm{sm}$ & No significativo & Ib \\
Lavigne $(2002)^{42}$ & Budesonida intrasinusal & $3 \mathrm{sm}$ & Mejoría síntomas & Ib \\
Lund $(2004)^{43}$ & Budesonida & $20 \mathrm{sm}$ & Mejoría síntomas & Ib \\
\hline
\end{tabular}

sm: semanas. Ib: laboratorio. 
RSC. Los corticoides inhalados son capaces de abordar la respuesta inflamatoria subyacente a la congestión nasal, obstrucción y dolor facial (entre los síntomas más angustiantes de la rinosinusitis) y promueven el drenaje. Los corticoides inhalados se han recomendado como tratamiento de primera línea, debido a su capacidad de mejorar los síntomas y resultados clínicos si se administran concomitantemente con antibióticos ${ }^{42}$.

\section{Antihistamínicos}

Los antihistamínicos son medicamentos de amplio uso en la práctica clínica, particularmente en RSCCP52. Sin embargo, no existe evidencia para sostener algún rol de los antihistamínicos en esta patología. Es así como un estudio realizado a 45 pacientes con diagnóstico de poliposis nasal residual o recurrente posetmoidectomía comparó el tratamiento con cetirizina (20 mg 2 veces al día) con placebo durante 3 meses. Los autores observaron que el número y tamaño de los pólipos se mantuvo sin cambios durante el periodo de estudio. Se encontró, además, que la cetirizina reducía eficazmente estornudos y rinorrea. También logró un efecto beneficioso sobre la obstrucción nasal. Los efectos secundarios con $20 \mathrm{mg}$ de cetirizina, el doble de la dosis diaria recomendada para adultos, fueron pocos y comparables con placebo ${ }^{53}$.

Se puede concluir que el uso de antihistamínicos en pacientes con diagnóstico de RSCCP sólo es efectivo en manejar los síntomas derivados de la alergia, sin mostrar efectos en los pólipos, recomendándose su uso sólo en casos en los que la poliposis se asocie a sintomatología alérgica.

\section{Antifúngicos}

El rol del material fúngico inhalado en el desarrollo de procesos inflamatorios de la vía aérea superior fue un tema muy debatido durante la década de los '90. Como extensión de esta teoría, se adjudicó a las hifas fúngicas responsabilidad en la RSC, por lo que se usó terapia antifúngica oral y tópica para manejo de estos pacientes.

Diversos estudios han tratado de demostrar la utilidad del uso de anfotericina $B$ en el tratamiento de la rinosinusitis crónica. Ponikau y cols ${ }^{54}$ demostraron una mejoría del $75 \%$ en parámetros clínicos y endoscópicos en pacientes con RSCSP sometidos a tratamiento con lavados nasales con anfotricina B. Sin embargo este mismo autor, años más tarde, desarrolló un estudio clínico controlado, en el que se incluyeron 30 pacientes con RSCSP, no observando grandes diferencias entre el grupo tratado con anfotericina y el grupo placebo luego de 6 meses ${ }^{55}$.

Hallazgos similares fueron encontrados por Weschta y $\operatorname{cols}^{56}$ quienes diseñaron un estudio clínico controlado en el que participaron 60 pacientes con RSCCP, donde tampoco encontraron beneficio significativo con el uso de anfotericina $B$, por un período de 8 semanas.

Intentando aclarar el rol de los antifúngicos intranasales en el tratamiento de la RSC, Ebbens y cols $^{57}$ evaluaron la efectividad del uso de lavados nasales con anfotericina al compararlo con lavados nasales con placebo, en pacientes con RSCCP y RSCSP. Seleccionaron al azar un total de 166 pacientes con RSC para infusión de $25 \mathrm{ml}$ de anfotericina $B(100 \mathrm{mg} / \mathrm{ml})$ o placebo (en cada fosa nasal dos veces al día durante 3 meses). Los resultados, después de 13 semanas de tratamiento, no evidenciaron diferencias estadísticamente significativas entre ambos grupos respecto a reducción del puntaje de la escala visual análoga y de endoscopía nasal, como resultados principales, ni en flujo inspiratorio máximo nasal, características de pólipos, calidad de vida ni escala visual análoga individual. Concluyeron que los lavados nasales con anfotericina B no reducen los signos ni síntomas clínicos en pacientes con RSC, por lo que no recomiendan su uso tanto en RSCCP como en RSCSP.

Esto fue reafirmado en reciente estudio clínico controlado en el que se demostró que el uso de irrigación con anfotericina $B$ en pacientes con RSCSP no produce mejoría significativa de síntomas ni hallazgos endoscópicos en comparación con suero salino $0^{58}$.

Por otra parte, también se ha evaluado el efecto de la terapia antifúngica oral. Kennedy y cols ${ }^{59}$ realizaron estudio clínico controlado, en el que se trataron 53 pacientes con terbinafina oral en altas dosis durante 6 semanas, no mostrándose mejoría en puntaje de síntomas ni de TC.

Por lo tanto, según la evidencia actual no parece haber un rol de los lavados con antifúngicos 
en el tratamiento de la RSCCP y RSCSP. La evidencia disponible se resume en la Tabla 11 15-57,59.

\section{Descongestionantes}

En teoría, tanto los descongestionantes tópicos como los sistémicos tienen un efecto antiinflamatorio al disminuir los niveles de óxido nítrico sintetiza y por acción antioxidante, que podría ser beneficiosa; sin embargo, no existen estudios clínicos controlados en RSC.

En estudio efectuado a una población pediátrica con diagnóstico de RSC maxilar en tratamiento médico, el uso de descongestionantes no mostró un efecto superior al uso de solución salina al evaluar el drenaje del $s e n 0^{60}$. En la actualidad no se recomienda el uso rutinario de descongestionantes en RSC.

\section{Lavado intranasal}

El lavado intranasal es de uso frecuente en pacientes con RSC por su bajo costo, alta accesibilidad y escasos efectos adversos asociados. Sin embargo, no existen estudios bien diseñados que analicen métodos de instilación, cantidad, frecuencia y concentración. Existen algunos que han demostrado mejoría en síntomas, calidad de vida, parámetros endoscópicos y de imágenes ${ }^{61-64}$.

En general, se prefiere usar solución hipertónica, porque tiene aparentemente buen efecto sobre el clearance mucociliar ${ }^{65,66}$.

En una revisión sistemática realizada por la base de datos Cochrane (2007) ${ }^{67}$ se evaluó el efecto del uso de irrigación salina nasal en la sintomatología de RSC. Fueron seleccionados 8 trabajos que cumplían criterios de inclusión. Tres estudios compararon solución salina con ausencia de tratamiento $0^{68,69,70}$, uno con placebo (reflexología) $)^{71}$, uno como terapia adyuvante ${ }^{72}$, y otro en comparación a corticoides inhalados ${ }^{73}$. Dos estudios ponderaron diferentes soluciones hipertónicas con solución salina isotónica ${ }^{74,75}$. Esta revisión concluyó señalando que existe evidencia respecto a que la solución salina es beneficiosa en el tratamiento de los síntomas de RSC cuando se utiliza ya sea en modalidad única de tratamiento o como terapia adyuvante, mejorando puntajes de síntomas y calidad de vida. No obstante, la solución salina no fue tan efectiva como los corticoides inhalados, demostrado al medir puntajes de calidad de vida.

Algunas evidencias sugieren que la solución salina hipertónica mejora parámetros objetivos (puntaje radiológico), pero su efecto sobre los síntomas es menos claro.

Es posible concluir que las soluciones salinas son bien toleradas, aunque los efectos secundarios menores son comunes; sin embargo, su acción beneficiosa parece superar los inconvenientes en la mayoría de los pacientes. Por consiguiente, el uso de solución salina tópica puede incluirse como tratamiento adyuvante de los síntomas de RSC.

\section{Antileucotrienos}

Los leucotrienos son mediadores inflamatorios que han sido involucrados en múltiples enfermedades del tracto respiratorio, como rinitis alérgica, asma y poliposis nasal. A raíz de lo anterior se ha estudiado el rol de sus antagonistas en el tratamiento de la RSCCP, el cual se basa, entre otros efectos, en la reducción del recuento de eosinófilos ${ }^{76,77}$.

Es así como en un estudio se observó que el uso de montelukast generaba una mejoría en síntomas subjetivos y recuentos de eosinófilos en pacientes con RSCCP y rinitis alérgica con tratamiento esferoidal tópico $0^{78}$.

Tabla 11. Antifúngicos en rinosinusitis crónica

\begin{tabular}{|lllc|}
\hline Estudio & Droga & Efectos & Nivel de evidencia \\
\hline Weschta $(2004)^{48}$ & Anfotericina B spray & Sin diferencias en TC y NFC & $\mathrm{lb}$ \\
Ponikau $(2005)^{47}$ & Anfotericina B lavado & Sin diferencia sintomáticas & $\mathrm{lb}$ \\
Kennedy $(2005)^{51}$ & Terbinafina oral/placebo & Sin diferencias & $\mathrm{lb}$ \\
Ebens $(2006)^{49}$ & Anfotericina B lavado & Sin diferencias & $\mathrm{lb}$ \\
\hline
\end{tabular}

TC: tomografía computarizada. NFC: nasofibroscopía. Ib: laboratorio. 
Por otra parte, Stewart y cols ${ }^{79}$ compararon el uso de montelukast con placebo, en el tratamiento de pacientes con RSCCP, quienes ya se encontraban en tratamiento esteroidal tópico crónico. Dicho estudio mostró un beneficio significativo con el uso de montelukast en comparación con placebo en síntomas como cefalea, dolor facial y estornudos, mejoría que perdura mientras se utiliza este fármaco.

Dhalen y cols $^{80}$ evaluaron individuos con poliposis nasal quienes, además, tenían intolerancia a la aspirina y asma bronquial, encontrando beneficio significativo en el uso de inhibidores de la 5-lipooxigenasa, en cuanto a la medición de flujo nasal y síntomas como anosmia, rinorrea y congestión nasal.

Por consiguiente, el uso de antagonistas de los leucotrienos parecería ser una alternativa útil en pacientes con RSCCP, particularmente en quienes, además, existe asma bronquial asociada. Sin embargo, esto debe ser respaldado por estudios clínicos controlados de mayor número a los existentes actualmente.

\section{Inhibidores de la bomba de protones}

El reflujo gastroesofágico (RGE) ha sido sugerido como causa de RSC en población pediátrica; sin embargo, su contribución a la patogenia de la RSC en adultos no ha sido estudiada de manera sistemática. No existen estudios clínicos controlados que muestren beneficio en el uso de inhibidores de la bomba de protones.

En un ensayo clínico realizado por Ulualp y cols ${ }^{81}$ se evaluó la contribución del RGE a la patogenia de la RSC en un grupo de pacientes no estudiados sistemáticamente comparándose con un grupo control sano. Mediante técnica de monitoreo de $\mathrm{pH}$ esófago-faríngeo ambulatoria se evaluaron episodios de reflujo, observándose que la prevalencia de reflujo faríngeo fue significativamente mayor en el grupo de pacientes con RSC que no responden a tratamiento convencional comparado con el grupo control. Estos hallazgos sugieren que el RGE podría contribuir a la patogénesis de la RSC en algunos pacientes adultos.

En cambio, en pacientes con RSCCP no poseería ningún rol demostrado.

\section{TRATAMIENTO MÉDICO MÁXIMO}

La RSC es una patología frecuente, de curso prolongado y que altera en forma importante la calidad de vida de quienes la padecen.

La gran mayoría de los pacientes logra responder sintomáticamente al tratamiento médico y sólo un porcentaje de ellos es candidato a manejo quirúrgico, principalmente por persistencia de síntomas, pese a contar con la terapia médica máxima disponible.

Tradicionalmente se ha usado el concepto de tratamiento médico máximo para definir el manejo óptimo preoperatorio, el cual debe construirse en base a la mejor evidencia disponible. Sin embargo, aún no existe consenso sobre el mejor tratamiento médico a utilizar. En relación a esto, existen distintos tratamientos que han demostrado cierta utilidad. En primer lugar, está el uso de los corticoides inhalados como herramienta terapéutica de primera línea en el manejo crónico, tanto de pacientes con rinosinusitis crónica con pólipos como aquellos sin pólipos. Estos, mediante su poderoso efecto antiinflamatorio logran disminuir el dolor facial, la congestión y obstrucción nasal.

Para aquellos pacientes con rinosinusitis crónica sin evidencia de alergia, ya sea clínica 0 de laboratorio, surge como alternativa novedosa y eficaz el uso de terapias antibióticas por tiempos prolongados. Particularmente el uso de macrólidos, los cuales utilizados en bajas dosis y por un tiempo de 3 meses, tienen la capacidad de acumularse en el interior de las células inflamatorias y desde ahí ejercer su efecto antiinflamatorio a distintos niveles. De este modo, tal efecto se prolonga en el tiempo.

No existe referencia en la literatura para el uso de antibióticos por cortos períodos de tiempo en el tratamiento de la RSC. Aparentemente su mayor utilidad está dada en el manejo de las exacerbaciones agudas en estos pacientes.

Por otra parte, se ha recomendado como complemento a lo anterior, el uso de lavados nasales, ya que son de bajo costo y se asocian a pocos y leves efectos adversos. Aún no está del todo claro si el suero hipertónico favorece la evolución de estos pacientes.

Sabemos que no es posible dar "recetas de cocina", debido a que cada caso debe analizarse en 
Tabla 12. Tratamiento médico máximo

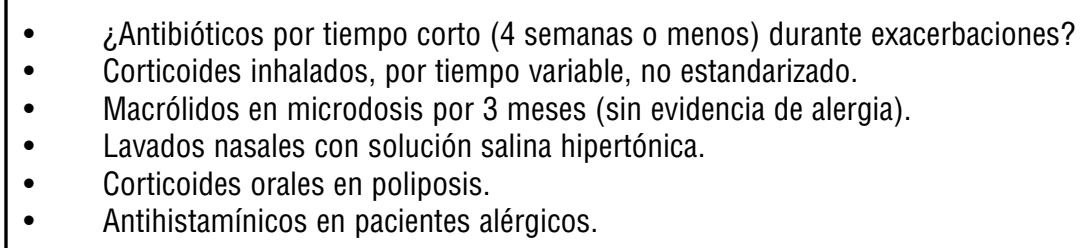

forma individual. Sin embargo existe consenso en terapias demostradas, las que se resumen en la Tabla 12.

Es así, que el diagnóstico adecuado es fundamental, y debe basarse en una minuciosa historia clínica, complementada con una evaluación endoscópica nasal y TC de cavidad nasal y senos paranasales. Debe incluirse además, estudio de inmunidad en todos los casos.

De este modo el conocimiento y uso racional del tratamiento médico nos permitirá elegir mejor a los candidatos quirúrgicos y así ofrecer a nuestros pacientes el mejor tratamiento disponible en cada caso.

\section{BIBLIOGRAFÍA}

1. Fokkens WJ, Lund VJ, Mullol J. et al. European Position Paper on Nasal Polyps 2007. Rhinology 45; 20 (suppl): 1-139.

2. Pearlman an, Conley DB. Review of current guidelines related to the diagnosis and treatment of rhinosinusitis.Curr Opin Otolaryngol Head Neck Surg. 2008; 16: 226-30.

3. Rosenfeld RM, Singer M, Jones S. Systematic review of antimicrobial therapy in patients with acute rhinosinusitis. Otolaryngol Head Neck Surg 2007; 137 (suppl): S32-45.

4. Meltzer eO, Hamilos DL, Hadley Ja. et al. Rhinosinusitis: Establishing definitions for clinical research and patient care. Otolaryngol Head Neck Surg. 2004; 131 (Suppl): S1-S62.

5. Bernardes JF, Shan J, Tewfik M, Hamid Q, Frenkiel S, EIdelman DH. Protein nitration in chronic sinusitis and nasal polyposis: Role of eosinophils. Otolaryngol Head Neck Surg 2004; 131: 696-703.
6. Claeys S, Be Belder T, Holtappels G, et al. Macrophage mannose receptor in chronic sinus disease. Allergy 2004; 59(6): 606-12.

7. Kramer MF, Burow G, Pfrogner E, Rasp G. In vitro diagnosis of chronic nasal inflammation. Clin Exp Allergy 2004; 34(7): 1086-92.

8. Rudack C, Sachse F, Alberty J. Chronic rhinosinusitis: Need for further classification? Inflamm Res 2004; 53(3): 111-7.

9. Polzehl D, Weschta M, Podielski A, Riechelmann H, RIMEK $D$. Fungus culture and PCR in nasal lavage samples of patients with chronic rhinosinusitis. $J$ Med Microbiol 2005; 54(1): 31-7.

10. Seiberling KA, Grammer L, Kern RC. Chronic rhinosinusitis and superantigens. Otolaryngol Clin North Am. 2005; 38: 1215-36.

11. Hafidh M, Harney M, Kane R, Donnelly M, Landers $R$, Sмутн $D$. The role of fungi in the etiology of chronic rhinosinusitis: A prospective study. Auris Nasus Larynx 2006; 26.

12. Van Zele $T$, Claeys $S$, Gevaert $P$, et al. Differentiation of chronic sinus diseases by measurement of inflammatory mediators. Allergy 2006; 61: 1280-9.

13. JankowskI R. Eosinophils in the pathophysiology of nasal polyposis. Acta Otolaryngol 1996; 116: 160-3.

14. LOESEL LS. Immunopathologic study of chronic sinusitis: a proposal for atopic and non-atopic lgE-activated mast cell allergic inflammation. Ann Otol Rhinol Laryngol 2001; 110(5 Pt 1): 447-52.

15. Sobol SE, Christodoulopoulos P, Manoukian JJ, et AL. Cytokine profile of chronic sinusitis in patients with cystic fibrosis. Arch Otolaryngol Head Neck Surg 2002; 128: 1295-8.

16. Shin SH, Lee SH, Jeong HS, Kita H. The effect of nasal polyp epithelial cells on eosinophil 
activation. Laryngoscope 2003; 113(8): 1374-7.

17. Conley DB, Tripathi A, Selberling KA, et al. Superantigens and chronic rhinosinusitis II: analysis of T-cell receptor $\mathrm{V}$ beta domains in nasal polyps. Am J Rhinol 2006; 20(4): 451-5.

18. HaO J, Pang YT, Wang DY. Diffuse mucosal inflammation in nasal polyps and adjacent middle turbinate. Otolaryngol Head Neck Surg 2006; 134(2): 267-75.

19. Brazilian Guidelines on Rhinosinusitis. Rev Bras Otorrinolaringol [online] 2008; 2 (suppl): 6-59.

20. Lund VJ. Maximal medical therapy for chronic rhinosinusitis. Otolaryngol Clin North $\mathrm{Am}$ 2005; 38(6): 1301-10.

21. Legent F, Bordure P, Beauvillain C, et al. A double-blind comparison of ciprofloxacin and amoxycillin/clavulanic acid in the treatment of chronic sinusitis. Chemotherapy 1994; 40(suppl 1): 8-15.

22. McNally PA, White MV, Kaliner MA. Sinusitis in an allergist's office: Analysis of 200 consecutive cases. Allergy Asthma Proc 1997; 18(3): 169-75.

23. Subramanian HN, Schechtman KB, Hamilos DL. A retrospective analysis of treatment outcomes and time to relapse after intensive medical treatment for chronic sinusitis. Am J Rhinol 2002; 16(6): 303-12.

24. Namyslowski G, Misiolek M, Czecior E, et al. Comparison of the efficacy and tolerability of amoxycillin/clavulanic acid $875 \mathrm{mg}$ b.i.d. with cefuroxime $500 \mathrm{mg}$ b.i.d. in the treatment of chronic and acute exacerbation of chronic sinusitis in adults. J Chemother 2002; 14(5): 50817.

25. Gold SM, TAMI TA. Role of middle meatus aspiration culture in the diagnosis of chronic sinusitis. Laryngoscope 1997; 107(12 Pt 1): 1586-9.

26. Klossek JM, Dubreuil L, Richet H, Richet B, BeUtTer P. Bacteriology of chronic purulent secretions in chronic rhinosinusitis. $J$ Laryngol Otol 1998; 112(12): 1162-6.

27. Vogan JC, Bolger WE, Keyes AS. Endoscopically guided sinonasal cultures: A direct comparison with maxillary sinus aspirate cultures. Otolaryngol Head Neck Surg 2000; 122: 370-3.

28. Talbot GH, Kennedy DW, Scheld WM, Granito K. Rigid nasal endoscopy versus sinus puncture and aspiration for microbiologic documentation of acute bacterial maxillary sinusitis. Clin Infect Dis 2001; 33(10): 1668-75.

29. Joniau S, Vlaminck $S$, Van Landuyt H, Kuhweide R, Dıck C. Microbiology of sinus puncture versus middle meatal aspiration in acute bacterial maxillary sinusitis.Am $J$ Rhinol 2005; 19(2): 135-40.

30. Kudoh S, Azuma A, Yamamoto M, Izumi T, Ando M. Improvement of survival in patients with diffuse panbronchiolitis treated with low-dose erythromycin. Am J Respir Crit Care Med 1998; 157(6 Pt 1): 1829-32.

31. SaIman L. The use of macrolide antibiotics in patients with cystic fibrosis. Curr Opin Pulm Med 2004; 10 (6): 515-23.

32. Hirakata Y, Kaku M, Mizukane R, et al. Potential effects of erythromycin on host defense systems and virulence of Pseudomonas aeruginosa. Antimicrob Agents Chemother 1992; 36(9): 1922-7.

33. Cervin A, Wallwork B. Anti-inflammatory effects of macrolide antibiotics in the treatment of chronic rhinosinusitis. Otolaryngol Clin North Am 2005; 38: 1339-50.

34. HASHIBA M, BaBA S. Efficacy of long-term administration of clarithromycin in the treatment of intractable chronic sinusitis. Acta Otolaryngol 1996; 525 (suppl): 73-8.

35. Wallwork B, Coman W, Mackay-Sim A, Greiff L, Cervin A. A double-blind, randomized, placebocontrolled trial of macrolide in the treatment of chronic rhinosinusitis. Laryngoscope 2006; 116(2): 189-93.

36. Nishi K, Mizuguch M, Tachibana H, et al. Effect of clarithromycin on symptoms and mucociliary transport in patients with sino-bronchial syndrome. Nihon Kyobu Shikkan Gakkai Zasshi 1995; 33 (12): 1392-400.

37. Ragab S, Lund V, Sccading G. Evaluation of the medical and surgical treatment of chronic rhinosinusitis: a prospective, randomized, controlled trial. Laryngoscope 2004; 114(5): 923-30.

38. Van Camp C. Results of oral steroid treatment in nasal polyposis. Rhinology. 1994; 32(1): 5-9.

39. Damm M. Effects of systemic steroid treatment in chronic polypoid rhinosinusitis evaluated with magnetic resonance imaging. Otolaryngol Head Neck Surg 1999; 120(4): 517-23. 
40. Benítez P. A short course of oral prednisone followed by intranasal budesonide is an effective treatment of severe nasal polyps. Laryngoscope 2006; 116(5): 770-5.

41. Hissaria P. Short course of systemic corticosteroids in sinonasal polyposis: a double-blind, randomized, placebo-controlled trial with evaluation of outcome measures. $J$ Allergy Clin Immunol 2006; 118(1): 128-33.

42. Lund V. Therapeutic targets in rhinosinusitis: Infection or inflammation? Medscape J Med 2008; 10(4): 105.

43. KEITH P. Efficacy and tolerability of fluticasone propionate nasal drops 400 microgram once daily compared with placebo for the treatment of bilateral polyposis in adults. Clin Exp Allergy 2000; 30 (10): 1460-8.

44. PentTilä M. Dose-related efficacy and tolerability of fluticasone propionate nasal drops 400 microg once daily and twice daily in the treatment of bilateral nasal polyposis: A placebo-controlled randomized study in adult patients. Clin Exp Allergy 2000; 30(1): 94-102.

45. SMALL CB. Efficacy and safety of mometasone furoate nasal spray in nasal polyposis. J Allergy Clin Immunol 2005; 116(6): 1275-81. Epub 2005 Sep 26.

46. StJÄRNE P. A randomized controlled trial of mometasone furoate nasal spray for the treatment of nasal polyposis. Arch Otolaryngol Head Neck Surg 2006; 132(2): 179-85.

47. Cuenant G. Efficacy of endonasal neomycintixocortol pivalate irrigation in the treatment of chronic allergic and bacterial sinusitis. ORL $J$ Otorhinolaryngol Relat Spec 1986; 48(4): 226-32.

48. SYKES DA. Relative importance of antibiotic and improved clearance in topical treatment of chronic mucopurulent rhinosinusitis. A controlled study. Lancet 1986; 16; 2(8503): 359-60.

49. PARIKH A. Topical corticosteroids in chronic rhinosinusitis: A randomized, double-blind, placebo-controlled trial using fluticasone propionate aqueous nasal spray. Rhinology 2001; 39(2): 75-9.

50. LAVIGNE F. Intrasinus administration of topical budesonide to allergic patients with chronic rhinosinusitis following surgery. Laryngoscope 2002; 112: 858-64.
51. Lund VJ. Efficacy and tolerability of budesonide aqueous nasal spray in chronic rhinosinusitis patients. Rhinology 2004; 42: 57-62.

52. Bhattacharyy NL. The economic burden and symptom manifestations of chronic rhinosinusitis. Am J Rhinol 2003; 17: 27-32.

53. Haye R, Aanesen JP, Burtin B, Donnelly F, Duby C.The effect of cetirizine on symptoms and signs of nasal polyposis. J Laryngol Otol 1998; 112(11): 1042-6.

54. Ponikau JU, Sherris DA, Kita H, Kern eB. Intranasal antifungal treatment in 51 patients with chronic rhinosinusitis. J Allergy Clin Immunol 2002; 110: 862-6.

55. Ponikau JU, Sherris da, Weaver A, Kita $H$. Treatment of chronic rhinosinusitis with intranasal amphotericin B: A randomized, placebo-controlled, double-blind pilot trial. $J$ Allergy Clin Immunol 2005; 115: 125-31.

56. Weschta M, Rimek D, Formanek M, Polzehl D, Podbielski A, Riechelmann $H$. Topical antifungal treatment of chronic rhinosinusitis with nasal polyps: A randomized, double-blind clinical trial. J Allergy Clin Immunol 2004; 113: 1122-8.

57. EBBENS F. Amphotericin B nasal lavages: Not a solution for patients with chronic rhinosinusitis. J Allergy Clin Immunol 2006; 118: 1149-56.

58. Kal-LI LIANG, M.D. Amphotericin B irrigation for the treatment of chronic rhinosinusitis without nasal polyps: A randomized, placebocontrolled, double-blind study. Am J Rhinol 2008; 22: 52-58.

59. Kennedy DW, Kuhn FA, Hamilos DL, et al. Treatment of chronic rhinosinusitis with high-dose oral terbinafine: A double blind, placebo-controlled study. Laryngoscope 2005; 115: 1793-9.

60. OtTEN FW. Conservative treatment of chronic maxillary sinusitis in children. Long-term follow-up. Acta Otorhinolaryngol Belg 1997; 51(3): 173-5.

61. Bachmann G, Hommel G, Michel OL. Effect of irrigation of the nose with isotonic salt solution on adult patients with chronic paranasal sinus disease. Eur Arch Otorhinolaryngol 2000; 257(10): 537-41.

62. Taccariello M, Parikh A, Darby Y, et al. Nasal douching as a valuable adjunct in the management of chronic rhinosinusitis. Rhinology 1999; 37(1): 29-32. 
63. Rabago D, Zgierska A, Mundt M, et al. Efficacy of daily hypertonic saline nasal irrigation among patients with sinusitis: a randomized controlled trial. J Fam Pract 2002; 51(12): 1049-55.

64. Shoseyov D, Bibi H, Shal P, et AL. Treatment with hypertonic saline versus normal saline nasal wash of pediatric chronic sinusitis. J Allergy Clin Immunol 1998; 101(5): 602-5.

65. TALBOT ET AL. Mucociliary clearance and buffered saline hypertonic solution. Laryngoscope 1997; 197: 500-3.

66. Shoseyov D, Bibi H, Shai P, Shoseyov N, Shazberg G, HuRvitz H. Treatment with hypertonic saline versus normal saline nasal wash of pediatric chronic sinusitis. J Allergy Clin Immunol 1998; 101: 602-5.

67. Harvey R, Hannan SA, Badia L, Scadding G. Nasal saline irrigations for the symptoms of chronic rhinosinusitis. Cochrane Database of Systematic Reviews 2007, Issue 3. Art. No.: CD006394. DOI: 10.1002/14651858.CD006394.pub2.

68. Garavello W, Romagnoli M, Sordo L, Gaini RM, Di Berardino C, Angrisano A. Hypersaline nasal irrigation in children with symptomatic seasonal allergic rhinitis: A randomized study. Pediatr Allergy Immunol 2003; 14(2): 140-3.

69. Garavello W, Di Berardino F, Romagnoli M, Sambataro G, Gainl RM. Nasal rinsing with hypertonic solution: An adjunctive treatment for pediatric seasonal allergic rhinoconjunctivitis. Int Arch Allergy Immunol 2005; 137(4): 310-4.

70. Rabago D, Zgierska A, Mundt M, Barrett B, Bobula J, MaberRy R. Efficacy of daily hypertonic saline nasal irrigation among patients with sinusitis: A randomized controlled trial. J Fam Pract 2002; 51: 1049-55.

71. Heatley DG,McConnell KE, Kille TL, et al. Nasal irrigation for the alleviation of sinonasal symptoms. Otolaryngol Head Neck Surg 2001; 125: 44-8.

72. Rogkakou A, Guerra L, Massacane P, et al. Effects on symptoms and quality of life of hypertonic saline nasal spray added to antihistamine in persistent allergic rhinitis: A randomized controlled study. Allerg Immunol (Paris) 2005; 37: 353-6.

73. Cordray S, Harjo JB, Miner L. Comparison of intranasal hypertonic Dead Sea saline spray and intranasal aqueous triamcinolone spray in seasonal allergic rhinitis. Ear Nose Throat $J$ 2005; 84: 426-30.

74. Bachmann G, Hommel G, Michel 0. Effect of irrigation of the nose with isotonic salt solution on adult patients with chronic paranasal sinus disease. Eur Arch Otorhinolaryngol 2000; 257: 537-41.

75. Shoseyov D, Bibi H, Shai P, Shoseyov N, Shazberg G, HuRvitz $H$. Treatment with hypertonic saline versus normal saline nasal wash of pediatric chronic sinusitis. J Allergy Clin Immunol 1998; 101: 602-5.

76. McCarTY MS. Potential new avenues of treatment for chronic rhinosinusitis: An antiinflammatory approach. Otolaryngol Clin North Am 2005; 38: 1351-65.

77. Amrol D, Murray JJ. Curr Alternative medical treatment strategies for chronic hyperplastic eosinophilic sinusitis. Curr Opin Otolaryngol Head Neck Surg 2005; 1: 55-9.

78. KiefF DA, Busaba NY. Efficacy of montelukast in the treatment of nasal polyposis. Ann Otol Rhinol Laryngol 2005; 114(12): 941-5.

79. Stewart Ra, Ram B, Hamilton G, Weiner J, Kane KJ. Montelukast as an adjunct to oral and inhaled steroid therapy in chronic nasal polyposis. Otolaryngol Head Neck Surg 2008; 139(5): 682-7.

80. Dahlén B, Nizankowska E, Szczeklik a et al. Benefits from adding the 5-lipoxygenase inhibitor zileuton to conventional therapy in aspirin-intolerant asthmatics. Am J Respir Crit Care Med 1998; 157: 1187-94.

81. Ulualp S0, Toohill RJ, Hoffmann R, et al. Possible relationship of gastroesophagopharyngeal acid reflux with pathogenesis of chronic sinusitis. Am J Rhinol 1999; 13(3): 197-202. 